\title{
The Usefulness of Diffusion Weighted and Contrast Enhanced Magnetic Resonance Imaging in Characterization of Inconclusive Ovarian Mass
}

\author{
Ghada Mohammed Abd El Razeq ${ }^{1}$, Mohammad A. M. Ahmed ${ }^{2 *}$ \\ ${ }^{1}$ Department of Radiodiagnosis, Qena Faculty of Medicine, South Valley University, Qena, Egypt \\ ${ }^{2}$ Department of Obstetrics \& Gynecology, Qena Faculty of Medicine, South Valley University, Qena, Egypt \\ Email: *drmohammada zsvu@gmail.com
}

How to cite this paper: El Razeq, G.M.A. and Ahmed, M.A.M. (2020) The Usefulness of Diffusion Weighted and Contrast Enhanced Magnetic Resonance Imaging in Characterization of Inconclusive Ovarian Mass. International Journal of Medical Physics, Clinical Engineering and Radiation Oncology, 9, 24-33.

https://doi.org/10.4236/ijmpcero.2020.91003

Received: November 29, 2019

Accepted: December 31, 2019

Published: January 3, 2020

Copyright $\odot 2020$ by author(s) and Scientific Research Publishing Inc. This work is licensed under the Creative Commons Attribution International License (CC BY 4.0).

http://creativecommons.org/licenses/by/4.0/

\begin{abstract}
Introduction: Ovarian cancer is the commonest reason for death in females due to gynecologic malignancy around the world. In contrast to other gynecologic tumours, the definitive diagnosis is accomplished days after of the index surgery by the histopathology. Preoperative assessment based on conventional MRI is not accurate. Information is expanding increasing about the ability of new MRI modalities to assess ovarian mass. Aim of the study: To assess the ability of dynamic contrast-enhanced MRI (DCE-MRI), and Diffusion-weighted image (DWI) to describe uncertain ovarian masses. Patients and Methods: This is a retrospective study. Patients were referred from radio-diagnosis department and gynecology department of Qena faculty of medicine hospitals, South Valley University. Patients had uncertain adnexal masses at ultrasound. Magnetic resonance examination was doneutilizing 1.5 Tesla machine. The protocol included T1WI, T2WI, T1WI following contrast, and DWI. Results: We included 44 patients with different forms of complex cystic and solid ovarian masses. The final pathology of the ovarian masses was 18 benign, 4 borderline, and 22 malignant. The sensitivity, specificity, positive predictive value, negative predictive value, and accuracy for DWI were $100 \%, 94.4 \%, 96.3 \%, 100 \%$, and $97.7 \%$ respectively. The performance of DWI was higher than the conventional MRI and DCE-MRI. Conclusion: DCE-MRI and DWI have accepted ability to recognize malignant ovarian mass.
\end{abstract}

\section{Keywords}

Ovarian, Contrast, Diffusion, MRI 


\section{Introduction}

Ovarian cancer is one of the commonest cancers in the world. It is the commonest cause of death in women due to gynecologic malignancy around the world. It has a 5 year survival of $40 \%$ [1]. In contrast to other gynecologic cancers, the definitive diagnosis is achieved days after of the index surgery. The diagnosis is by pathology. Preoperative diagnosis based on clinical, laboratory, radiological, and even frozen section is not free of errors [2]. Preoperative biopsy is not offered except when the planed treatment is neoadjuvant chemotherapy or in palliative settings [3].

Ultrasound is the first diagnostic imaging. Conventional Magnetic resonance imaging (MRI) uses T1 \& T2 signals. MRI has 76\% sensitivity and 97\% specificity for diagnosis of uncertain ovarian mass. DCE-MRI has a higher sensitivity and specificity of $81 \%$ \& $98 \%$ (DCE-MRI) [4].

DWI is another modality in MRI. It depends on the different microstructure, cellular density, and microvasculature of malignant tissue. In ordinary body structure, water molecules move freely. Water in malignant tissue has restricted ability to diffuse. The apparent diffusion coefficient (ADC) value is a quantitative measure of diffusion. The computer unit in the MRI machine changes these quantities into a user-friendly qualitative image. This is the DWI. Tissues with limited diffusion are white on DWI and hypointense on the ADC map [5].

Earlier reports on the ability of DWI to recognize malignant ovarian tumour have found that DWI is not useful [6] [7]. Later reports found that DWI is useful in discrimination between benign and malignant ovarian mass [8] [9] [10]. A more recent study found a sensitivity of $84 \%$, and a specificity of $89 \%$ [ 11 ].

Information is expanding on the diagnostic ability of DCE-MRI and DWI. In this study, we expect to evaluate the ability of DCE-MRI, and DWI to describe uncertain ovarian masses.

Aim of the study: to assess the ability of dynamic contrast-enhanced MRI (DCE-MRI), and Diffusion-weighted image (DWI) to describe uncertain ovarian masses.

\section{Patients and Methods}

Design: a comparative study.

Patients: Patients were recruited from radio-diagnosis department \& gynecology department of Qena faculty of medicine hospitals, South Valley University. The study started from $1^{\text {st }}$ of July 2018 to $30^{\text {th }}$ of July 2019.

Patients had inconclusive adnexal masses at ultrasound. Masses satisfy any of the following criteria: 1) solid; 2) complex (solid and cystic); or 3) cystic with papillary projections or septations. Masses that obviously determined by ultrasound were out of the research.

Ethical considerations: written consents were obtained from participants. Patient data were anonymized. The ethical committee of research in our college approved the study. 


\section{Methods}

We did transabdominal ultrasound and transvaginal ultrasound for all cases. We used GE Logic P6 ultrasound machine (GE Healthcare Medical system; USA). We pursue the International Ovarian Tumor Analysis (IOTA) rules to characterize ovarian mass [12]. MR assessment was done at the magnetic resonance unit, Qena faculty of medicine Hospital, South Valley University. We used 1.5 Tesla amachine with body coil as a transmitter and a receiver of radio frequency signals (Achieva, Philips Medical System; Netherland). The MR assessment included T1WI, T2WI, post-contrast fat-suppressed T1WI, and DWI. DWI was done at b0, b500, b1000. Descriptive analysis was done. Data from the MR assessment included the mean size of the cyst or mass, the ADC value, and the morphologic criteria suggesting malignancy. We had executed an individual analysis for conventional MRI, DCE-MRI and DWI concerning their diagnostic performance in the characterization of ovarian masses/cysts. Masses are sent for histopathology after operations.

\section{Results}

We investigated 50 patients with 50 adnexal lesions. Six masses were omitted, three of them due to loss of follow up. Unfortunately, we excluded one mass because of lack of pathology (patient died before operation). Two masses are excluded, as they were broad-ligamentary fibroid. The remaining 44 patients with 44 complex ovarian masses were included in our study.

The patient's age ranged from 20 to 78 years old (mean 43.56 years). The main complaint was abdominal pain and/or abdominal distension; other cases came with different symptoms as subfertility or irregular vaginal bleeding.

The histopathology of the assessed masses were 18 benign, 4 borderline, and 22 malignant. The age range for patients with benign tumors was 20 - 65 years (mean $39 \pm 13$ years), while those with malignant tumors, their age range was 21 - 78 years (mean $46 \pm 16.953$ years).

Benign masses included six serous cystadenoma, five mucinous cysadenoma, three mature cystic teratoma, two ovarian fibroma, and fibrothecoma, and one tubo-ovarian abscess. There were four Borderline tumors (two serous and two mucinous). There were 22 invasive malignant masses (seven Serous cyst-adenocarcinoma, five Mucinous cyst-adenocarcinoma, three Metastatic krukenburg, three Immature teratoma, two fibrosarcoma, and two clear cell carcinoma).

The analysis of the size of the lesions is appeared in Table 1. The malignant and borderline ovarian lesions were bigger than the benign lesions.

The complex ovarian masses showed variable signal intensities in T1 and T2 as follows:

For T1 signal intensity, 34 of the included ovarian masses showed is o-intense signal intensity, 2 masses showed bright signals, while 8 masses showed mixed signal intensities. For T2 signal intensity, 32 of the masses showed high signals 
Table 1. Analysis of the ovarian lesions size.

\begin{tabular}{cccc}
\hline Dimension & Benign & Borderline & Malignant \\
\hline Minimum & $4.5 \mathrm{~cm}$ & $6 \mathrm{~cm}$ & $7 \mathrm{~cm}$ \\
Maximum & $15 \mathrm{~cm}$ & $22 \mathrm{~cm}$ & $25 \mathrm{~cm}$ \\
Mean \pm SD & $9.7 \pm 3.3$ & $14 \pm 7.3$ & $13.7 \pm 5.08$ \\
\hline
\end{tabular}

and 12 masses showed mixed signals (Figures 2-5). ADC values of malignant tumors showed a minimum of $0.7 \times 10^{-3} \mathrm{~mm}^{2} / \mathrm{s}$ and a maximum of $1.2 \times 10^{-3}$ $\mathrm{mm}^{2} / \mathrm{s}$. The mean $( \pm \mathrm{SD})$ was $1.01 \times 10^{-3} \mathrm{~mm}^{2} / \mathrm{s}( \pm 0.34)$, while ADC values of the benign masses showed a minimum of $1.2 \times 10^{-3} \mathrm{~mm}^{2} / \mathrm{s}$ and maximum of $2 \times$ $10^{-3} \mathrm{~mm}^{2} / \mathrm{s}$ with mean \pm SD $1.6 \times 10^{-3} \mathrm{~mm}^{2} / \mathrm{s}( \pm 0.27)$. The mean ADC value in benign tumors was obviously higher than that in malignant ones (Figures 1-5 \& Table 2).

For the sake of statistical evaluation, we incorporated the borderline tumors into the classification of malignant tumors. The histopathological reported 26 malignant and 18 benign lesions. The performance of the preoperative imaging is outlined in Table 3. The sensitivity, specificity, positive predictive value, negative predictive value, and accuracy for DWI were 100\%, 94.4\%, 96.3\%, 100\%, and 97.7\% respectively. The performance of DWI was higher than the conventional MRI and DCE-MRI. Borderline and malignant ovarian tumors showed restricted diffusion. Benign tumors had facilitated diffusion (Figures 2-5).

\section{Discussion}

Ovarian carcinoma has the most elevated death rate among tumours of the female genital tract. Differentiation between benign and malignant ovarian tumor is vital to offer the appropriate plan for each case [3] [13].

Conventional MRI assesses morphologic criteria of the lesion, such as wall thickening, intra luminal papilla, mural nodules, thick septae, and signal intensity on T1WI and T2WI. None of these criteria can consistently segregate benign from malignant lesions [4]. Development of novel MRI modalities like DCE MRI and DWI improves the diagnostic performance of MRI [4].

We had executed an individual analysis for conventional MRI, DCE-MRI and DWI concerning their diagnostic performance in the characterization of ovarian masses/cysts. We found that conventional MRI had $88.5 \%$ sensitivity and $72.2 \%$ specificity. This looks well with a meta-analysis of the value of MRI in characterization of ovarian mass/cyst in women with non-conclusive ultrasound evaluation. They found that the sensitivity and specificity was $76 \%$ and $97 \%$, respectively [14].

We found that DCE-MRI had $92.3 \%$ sensitivity and $88.8 \%$ specificity. This compares favourably to conventional MRI in our study. So, adding DCE to the MRI increased the accuracy of examination. Systematic review showed that DCE-MRI has $81 \%$ sensitivity and $98 \%$ specificity [14]. However, a more recent study showed $83 \%$ sensitivity and $75 \%$ specificity [15]. Malignant masses showed 


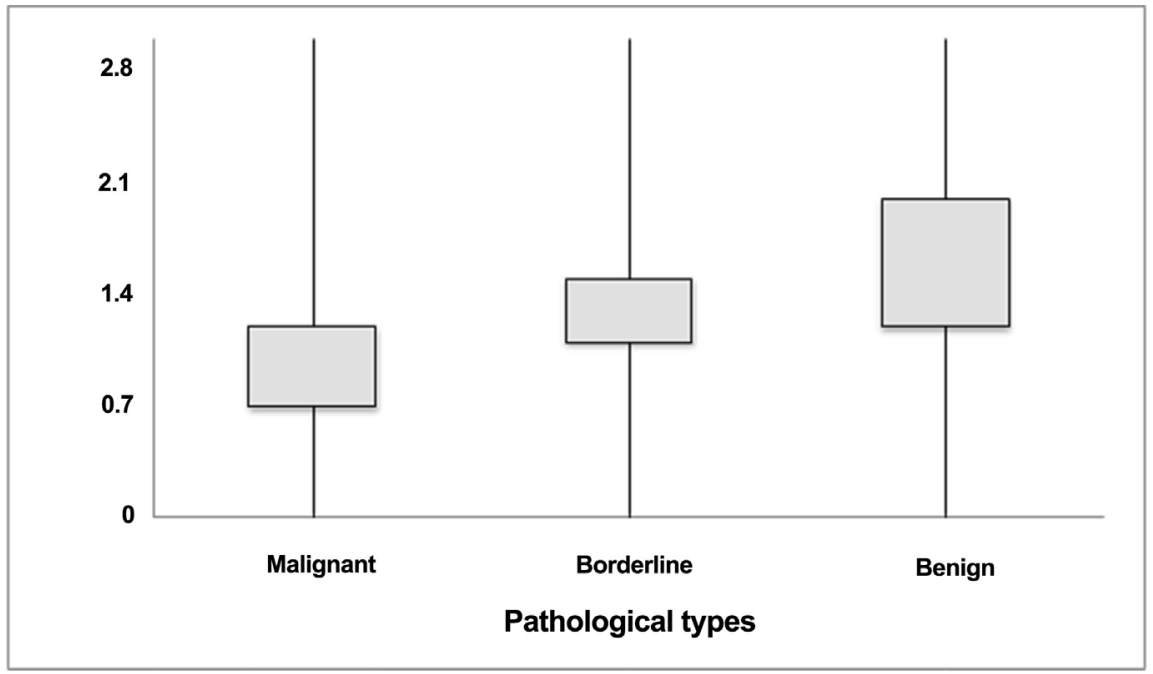

Figure 1. ADC Values $\left(10^{-3} / \mathrm{mm}^{2} / \mathrm{s}\right)$ of pathological types of ovarian lesions included in the study.
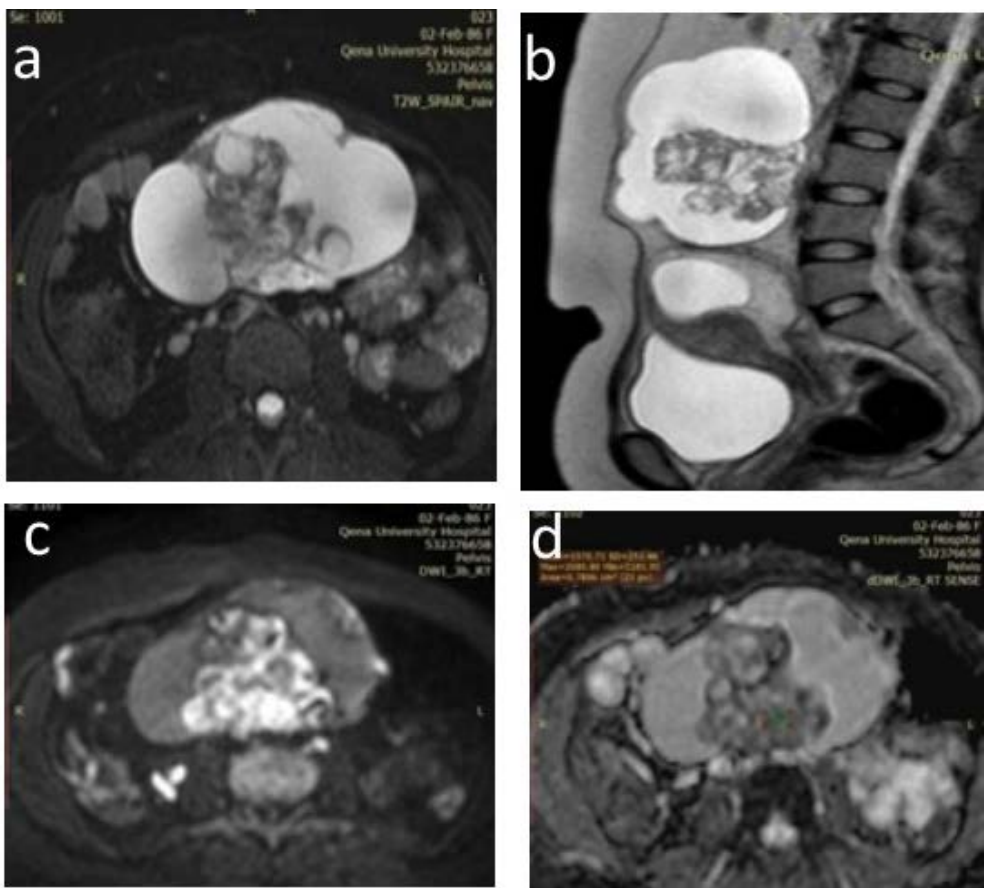

Figure 2. Female patient 32 years old presented by midline pelvic adnexal complex cystic lesion (arrow). (a) \& (b) MRI axial \& sagittal T2 shows midline pelvic unilocular complex cystic lesion with thick septations \& papillary soft tissue mass lesion inside. (c) DWI shows restricted solid component. (d) ADC map shows low ADC value $0.7 \times 10^{-3} \mathrm{~mm}^{2} / \mathrm{sec}$. Left adnexal histopathologically, proved mature cystic teratoma.

more intense enhancement than benign lesions. Difference was clearer in the early phase of the contrast study than the late phase [16] [17].

Our analysis revealed that DWI has $100 \%$ sensitivity, $94.4 \%$ specificity, $96.3 \%$ PPV, 100\% NPV, and 97.7\% accuracy. The performance of DWI was higher than conventional MRI and DCE-MRI. We found that all malignant lesions and one 

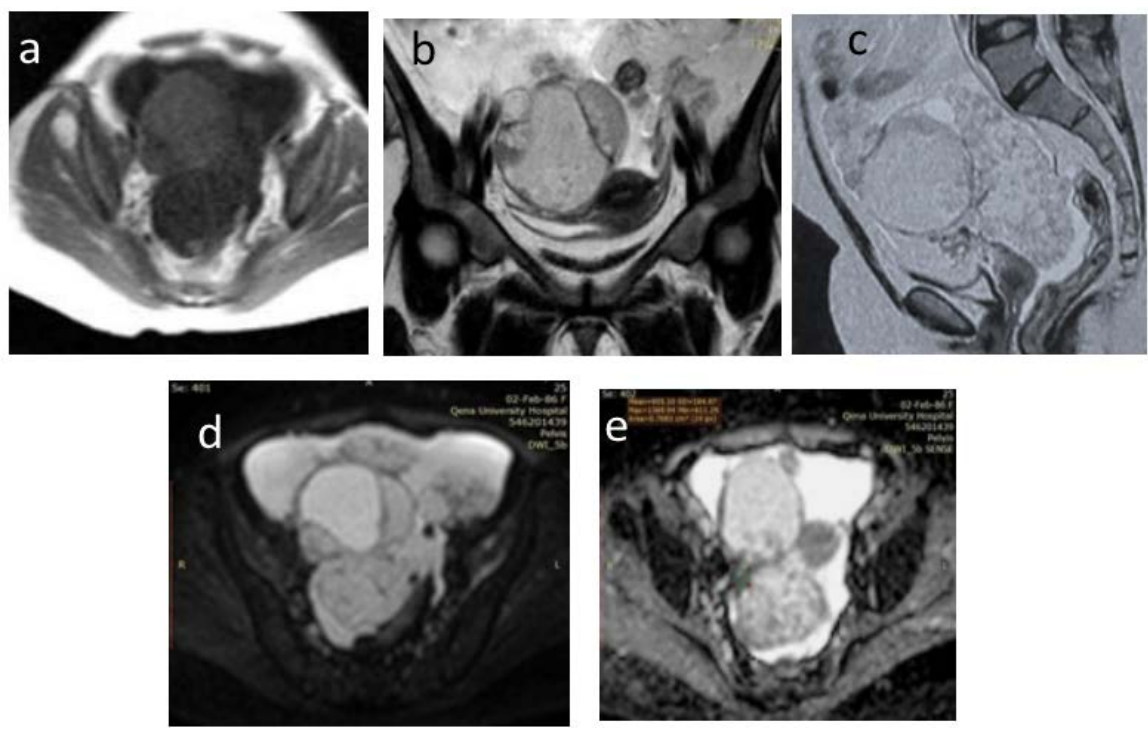

Figure 3. Female patient 38 years old presented by abdominal pain, pelvic US shows complex cystic right adnexal lesion with moderate ascites. (a) (b) \& (c) MRI axial T1, coronal \& sagittal T2 shows right ovarian multilocular complex cystic lesion (arrow)with thick septations\& peripheral soft tissue nodule inside, moderate ascites \& omental thickening. (d) DWI shows restricted cystic fluid contents. (e) ADC map shows low ADC value $0.9 \times 10^{-3} \mathrm{~mm}^{2} / \mathrm{sec}$. Papillary serous cystadenocarcinomais histologically proved.
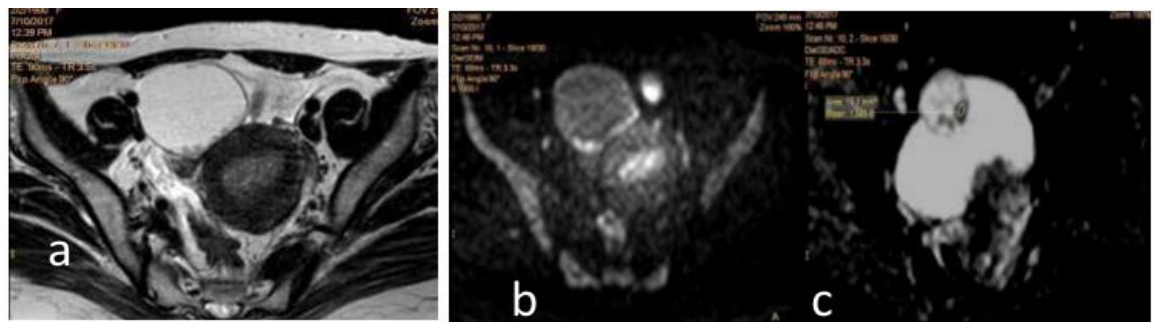

Figure 4. Female patient 45 years old presented by meno-metrorrhagia, pelvic US shows right ovarian cyst with mural nodule \& mild ascites. (a) MRI axial T2 shows right ovarian unilocular cystic lesion with peripheral nodular thickening, mild ascites; (d) DWI shows restricted soft tissue nodular thickening; (e) ADC map shows high ADC value $1.2 \times 10^{-3}$ $\mathrm{mm}^{2} / \mathrm{sec}$. Right ovarian borderline serous neoplasm is histologically proved.

case of dermoid cyst demonstrated a high signal on DWI. This may be ascribed to keratinized substance in dermoid cyst. These results are consistent with the conclusions in the previous researches. They showed that most of the malignant ovarian masses and some of the dermoid cysts had high intensity on DWI. Most of the benign lesions had low signal intensity on DWI [6] [18] [19].

In our study, the mean ADC values for malignant lesions were $1.01 \times 10^{-3} \pm$ $\left.0.34 \mathrm{~mm}^{2} / \mathrm{s}\right)$. The mean ADC values for benign lesions were $\left(1.6 \times 10^{-3} \pm 0.27\right.$ $\mathrm{mm}^{2} / \mathrm{s}$ ). Our cut-off value was $1.2 \times 10^{-3} \mathrm{~mm}^{2} / \mathrm{s}$. This agreed with findings by Takeuchi et al. They found the mean ADC value was $1.03 \times 10^{-3} \mathrm{~mm}^{2} / \mathrm{sin} \mathrm{ma}-$ lignant tumors and $1.38 \times 10^{-3} \mathrm{~mm}^{2} / \mathrm{s}$ in benign tumor [17]. A meta-analysis of 16 studies showed that DWI is able to distinguish between benign and malignant ovarian tumor with $91 \%$ sensitivity and $91 \%$ specificity [20]. 

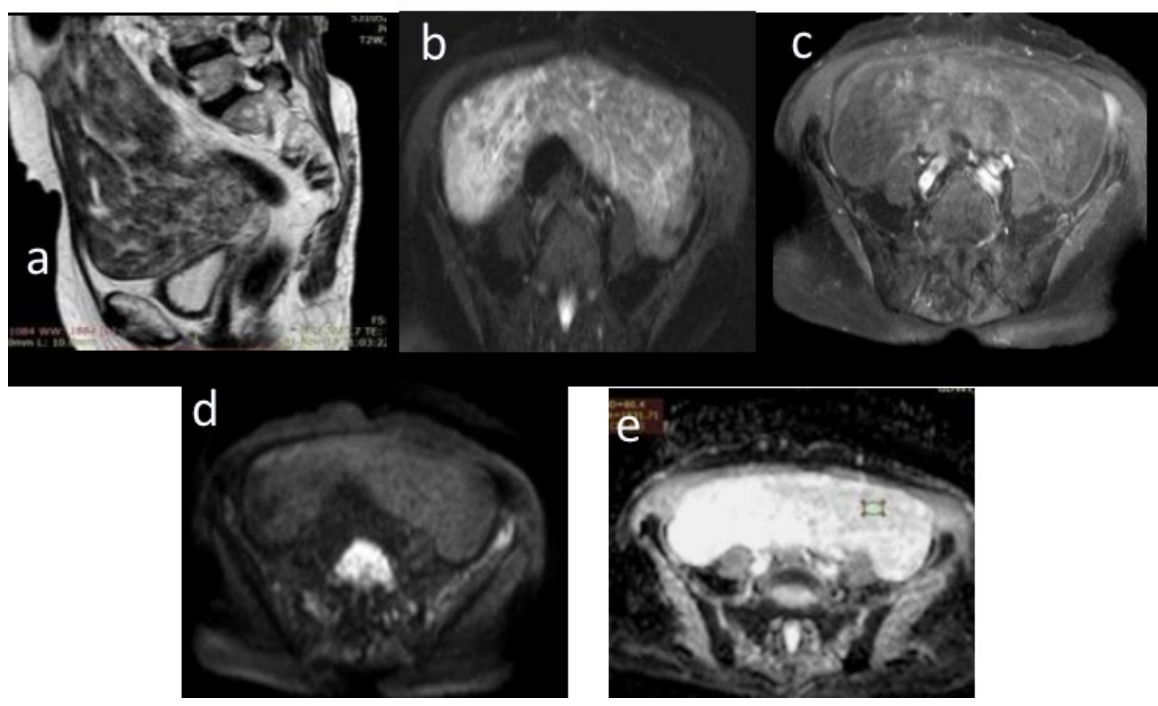

Figure 5. Female patient 60 years old presented by postmenopausal bleeding, pelvic US shows huge solid hypoechoic pelvic mass lesion. (a) MRI sagittal T2 shows huge anterior pelvic hypointense mass lesion displacing the uterus posteriorly with cystic endometrial thickening. (b) \& (c) T2 SPIR \& SPIR with contrast shows enhanced septae. (d) \& (e) DWI shows facilitated diffusion \& ADC map show high ADC value $2 \times 10^{-3} \mathrm{~mm}^{2} / \mathrm{sec}$. Ovarian fibroma is histologically proved.

Table 2. Showing the different ADC values of the included masses.

\begin{tabular}{|c|c|}
\hline Pathological type & ADV value (Range) \\
\hline BENIGN & $1.2-2 \times 10^{-3} \mathrm{~mm}^{2} / \mathrm{sec}$ \\
\hline Serous cystadenoma & $1.4-2 \times 10^{-3} \mathrm{~mm}^{2} / \mathrm{sec}$ \\
\hline Mucinous cystadenoma & $1.3-1.5 \times 10^{-3} \mathrm{~mm}^{2} / \mathrm{sec}$ \\
\hline Mature cystic teratoma & $1.2-1.5 \times 10^{-3} \mathrm{~mm}^{2} / \mathrm{sec}$ \\
\hline Ovarian fibroma & $1.6-1.8 \times 10^{-3} \mathrm{~mm}^{2} / \mathrm{sec}$ \\
\hline Fibrothecoma & $1.2 \times 10^{-3} \mathrm{~mm}^{2} / \mathrm{sec}$ \\
\hline Tubo-ovarian abscess & $1.3 \times 10^{-3} \mathrm{~mm}^{2} / \mathrm{sec}$ \\
\hline MALIGNANT & $0.7-1.2 \times 10^{-3} \mathrm{~mm}^{2} / \mathrm{sec}$ \\
\hline Serous cystadenocarcinoma & $0.7-1 \times 10^{-3} \mathrm{~mm}^{2} / \mathrm{sec}$ \\
\hline Mucinous cystadenocarcinoma & $0.9 \times 10^{-3} \mathrm{~mm}^{2} / \mathrm{sec}$ \\
\hline Metastatic Krukenberg & $1.2 \times 10^{-3} \mathrm{~mm}^{2} / \mathrm{sec}$ \\
\hline Clear cell carcinoma & $0.9 \times 10^{-3} \mathrm{~mm}^{2} / \mathrm{sec}$ \\
\hline Fibrosarcoma & $1.1 \times 10^{-3} \mathrm{~mm}^{2} / \mathrm{sec}$ \\
\hline Immature teratoma & $0.8-0.9 \times 10^{-3} \mathrm{~mm}^{2} / \mathrm{sec}$ \\
\hline Borderline serous neoplasm & $1.1-1.5 \times 10^{-3} \mathrm{~mm}^{2} / \mathrm{sec}$ \\
\hline Borderline mucinous neoplasm & $1.2 \times 10^{-3} \mathrm{~mm}^{2} / \mathrm{sec}$ \\
\hline
\end{tabular}


Table 3. The performance of the preoperative diagnosis.

\begin{tabular}{ccccc}
\hline & Ultrasound & Conventional MRI & DCE-MRI & DWI \\
\hline TP & 20 & 23 & 24 & 26 \\
FN & 6 & 3 & 2 & 0 \\
FP & 6 & 5 & 2 & 1 \\
TN & 12 & 13 & 16 & 17 \\
Sensitivity & $76.9 \%$ & $88.5 \%$ & $92.3 \%$ & $100 \%$ \\
Specificity & $66.6 \%$ & $72.2 \%$ & $88.8 \%$ & $94.4 \%$ \\
PPV & $76.9 \%$ & $82.1 \%$ & $85.7 \%$ & $96.3 \%$ \\
NPV & $66.6 \%$ & $81.2 \%$ & $88.8 \%$ & $100 \%$ \\
Accuracy & $72.2 \%$ & $81.8 \%$ & $90.9 \%$ & $97.7 \%$ \\
\hline
\end{tabular}

Limitations of the study: we did analysis of heterogonous group of tumors. Therefore, the absolute number of each tumour is small. Tumors have variable criteria according to their specific histologic composition. A larger study with focus on specific tumour type is needed.

\section{Conclusion}

DCE-MRI and DWI have accepted ability to distinguish between benign and malignant ovarian mass.

\section{Recommendations}

We recommend adding DCE-MRI and DWI to the conventional MRI in the assessment of ovarian mass. This will aid in accurate diagnosis. Further researches are needed to build up cumulative evidence of the value of novel signals of MRI.

\section{Authors' Approval}

Authors read and approve the manuscript. All authors have fulfilled the requirements for authorship. Each author believes that the manuscript represents honest work. This manuscript is not considered for publishing anywhere else.

\section{Conflicts of Interest}

We have no conflict of interest to disclose. We received no funding.

\section{References}

[1] Momenimovahed, Z., Tiznobaik, A., Taheri, S. and Salehiniya, H. (2019) Ovarian Cancer in the World: Epidemiology and Risk Factors. International Journal of Women's Health, 11, 287-299. https://doi.org/10.2147/IJWH.S197604

[2] American College of Obstetricians and Gynecologists' Committee on Practice Bulletins-Gynecology (2016) Practice Bulletin No. 174: Evaluation and Management of Adnexal Masses. Obstetrics and Gynecology, 128, e210-e226. https://doi.org/10.1097/AOG.0000000000001768 
[3] Armstrong, D.K., Alvarez, R.D., Bakkum-Gamez, J.N., Barroilhet, L., Behbakht, K., Berchuck, A., et al. (2019) NCCN Guidelines Insights: Ovarian Cancer, Version 1.2019. Journal of the National Comprehensive Cancer Network, 17, 896-909. https://doi.org/10.6004/jnccn.2019.0039

[4] Foti, P.V., Attinà, G., Spadola, S., Caltabiano, R., Farina, R., Palmucci, S., et al. (2016) MR Imaging of Ovarian Masses: Classification and Differential Diagnosis. Insights Imaging, 7, 21-41. https://doi.org/10.1007/s13244-015-0455-4

[5] Baliyan, V., Das, C.J., Sharma, R. and Gupta, A.K. (2016) Diffusion Weighted Imaging: Technique and Applications. World Journal of Radiology, 8, 785-798. https://doi.org/10.4329/wjr.v8.i9.785

[6] Fujii, S., Kakite, S., Nishihara, K., Kanasaki, Y., Harada, T., Kigawa, J., et al. (2008) Diagnostic Accuracy of Diffusion-Weighted Imaging in Differentiating Benign from Malignant Ovarian Lesions. Journal of Magnetic Resonance Imaging, 28, 1149-1156. https://doi.org/10.1002/jmri.21575

[7] Katayama, M., Masui, T., Kobayashi, S., Ito, T., Sakahara, H., Nozaki, A., et al. (2002) Diffusion-Weighted Echo Planar Imaging of Ovarian Tumors: Is It Useful to Measure Apparent Diffusion Coefficients? Journal of Computer Assisted Tomography, 26, 250-256. https://doi.org/10.1097/00004728-200203000-00015

[8] Mohaghegh, P. and Rockall, A.G. (2012) Imaging Strategy for Early Ovarian Cancer: Characterization of Adnexal Masses with Conventional and Advanced Imaging Techniques. Radiographics, 32, 1751-1773. https://doi.org/10.1148/rg.326125520

[9] Thomassin-Naggara, I., Darai, E., Cuenod, C.A., Fournier, L., Toussaint, I., Marsault, C., et al. (2009) Contribution of Diffusion-Weighted MR Imaging for Predicting Benignity of Complex Adnexal Masses. European Radiology, 19, 1544-1552. https://doi.org/10.1007/s00330-009-1299-4

[10] Zhang, P., Cui, Y., Li, W., Ren, G., Chu, C. and Wu, X. (2012) Diagnostic Accuracy of Diffusion-Weighted Imaging with Conventional MR Imaging for Differentiating Complex Solid and Cystic Ovarian Tumors at 1.5 T. World Journal of Surgical Oncology, 10, 237. https://doi.org/10.1186/1477-7819-10-237

[11] Garcia Prado, J., Gonzalez Hernando, C., Varillas Delgado, D., Saiz Martinez, R., Bhosale, P., Blazquez Sanchez, J., et al. (2019) Diffusion-Weighted Magnetic Resonance Imaging in Peritoneal Carcinomatosis from Suspected Ovarian Cancer: Diagnostic Performance in Correlation with Surgical Findings. European Journal of Radiology, 121, Article ID: 108696. https://doi.org/10.1016/j.ejrad.2019.108696

[12] Kaijser, J., Bourne, T., Valentin, L., Sayasneh, A., Van Holsbeke, C., Vergote, I., et al. (2013) Improving Strategies for Diagnosing Ovarian Cancer: A Summary of the International Ovarian Tumor Analysis (IOTA) Studies. Ultrasound in Obstetrics \& Gynecology, 41, 9-20. https://doi.org/10.1002/uog.12323

[13] Noone, A.-M., Cronin, K.A., Altekruse, S.F., Howlader, N., Lewis, D.R., Petkov, V.I., et al. (2017) Cancer Incidence and Survival Trends by Subtype Using Data from the Surveillance Epidemiology and End Results Program, 1992-2013. Cancer Epidemiology, Biomarkers \& Prevention, 26, 632-641. https://doi.org/10.1158/1055-9965.EPI-16-0520

[14] Iyer, V.R. and Lee, S.I. (2010) MRI, CT, and PET/CT for Ovarian Cancer Detection and Adnexal Lesion Characterization. AJR American Journal of Roentgenology, 194, 311-321. https://doi.org/10.2214/AJR.09.3522

[15] Li, H.M., Qiang, J.W., Ma, F.H. and Zhao, S.H. (2017) The Value of Dynamic Contrast-Enhanced MRI in Characterizing Complex Ovarian Tumors. Journal of Ova- 
rian Research, 10, 4. https://doi.org/10.1186/s13048-017-0302-y

[16] Sohaib, S.A.A. and Reznek, R.H. (2007) MR Imaging in Ovarian Cancer. Cancer Imaging, 7, S119-S129. https://doi.org/10.1102/1470-7330.2007.9046

[17] Nasr, E., Hamed, I., Abbas, I. and Khalifa, N.M. (2014) Dynamic Contrast Enhanced MRI in Correlation with Diffusion Weighted (DWI) MR for Characterization of Ovarian Masses. The Egyptian Journal of Radiology and Nuclear Medicine, 45, 975-985. https://doi.org/10.1016/j.ejrnm.2014.04.002

[18] Thomassin-Naggara, I., Toussaint, I., Perrot, N., Rouzier, R., Cuenod, C.A., Bazot, M., et al. (2011) Characterization of Complex Adnexal Masses: Value of Adding Perfusion- and Diffusion-Weighted MR Imaging to Conventional MR Imaging. Radiology, 258, 793-803. https://doi.org/10.1148/radiol.10100751

[19] van Nimwegen, L.W.E. and Mavinkurve-Groothuis, A.M.C. (2019) MR Imaging in Discriminating between Benign and Malignant Paediatric Ovarian Masses: A Systematic Review. European Radiology, 1-16.

https://doi.org/10.1007/s00330-019-06420-4

[20] Pi, S., Cao, R. and Qiang, J.W. (2018) Utility of DWI with Quantitative ADC Values in Ovarian Tumors: A Meta-Analysis of Diagnostic Test Performance. Acta Radiologica, 59, 1386-1394. https://doi.org/10.1177/0284185118759708 